\title{
Quantum information processing with atoms and photons
}

\section{Monroe}

FOCUS Center and D epartment of Physics, University of M ichigan, Ann Arbor, M ichigan 48109-1120, USA (e-mail: crmonroe@umich.edu)

Quantum information processors exploit the quantum features of superposition and entanglement for applications not possible in classical devices, offering the potential for significant improvements in the communication and processing of information. Experimental realization of large-scale quantum information processors remains a long-term vision, as the required nearly pure quantum behaviour is observed only in exotic hardware such as individual laser-cooled atoms and isolated photons. But recent theoretical and experimental advances suggest that cold atoms and individual photons may lead the way towards bigger and better quantum information processors, effectively building mesoscopic versions of 'Schrödinger's cat' from the bottom up.

n 1948, Claude Shannon discovered how to quantify information ${ }^{1}-$ a result so fundamental and revolutionary that in hindsight it is surprising it had not been formulated earlier. Shannon's bit, or binary digit, became the fundamental unit of information, providing a metric for comparing forms of information and optimizing the amount of resources needed to faithfully convey a given amount of information, even in the presence of noise.

Shannon'spioneering work notuncoincidentally prefaced theinformation age. Bitswerefound in nature, from unwieldy vacuum tubes in the 1940s to the modern semiconductor transistors of less than $10^{-5} \mathrm{~cm}$ in size. Under this impressive progression of technology, we have enjoyed an exponential growth in computing power and information processing speed given by the familiar 'M oore's Law', where computer chipshavedoubled in density every year or two. Unfortunately, the days (or years) of M oore's Law are numbered. As bits continually shrink in size, they will eventually approach the size of individual molecules - by theyear 2020 if the current growth continues. At thesenanometrelength scales, thelaws of quantum mechanics begin to hold sway. In fact, classical bits can still bestored and manipulated here, as verified by the ground-breaking theoretical work of Paul Benioff ${ }^{2,3}$ and Richard Feynman ${ }^{4}$ in the early 1980s, and foreseen in Feynman's remarkable 1959 charge towards nanotechnology, There's Plenty of Room at the Bottom 5 . But even if we could somehow reach the bottom, where quantum states of every atom in a computer chip host classical bits, therewould beno moreroom for further gainswithout splittingtheatom.

\section{Quantum rules}

If we instead focus on the pure quantum features of a collection of atom-sized bits, we indeed find more room. For an isolated quantum system, the fundamental unit of information is the quantum bit or 'qubit'. Qubits are just quantum two-level systems such as the spin of an electron or the polarization of a photon, and can be prepared in a coherent superposition state of 0 and 1 :

$|\Psi\rangle=\alpha|0\rangle+\beta|1\rangle$

Here, $\alpha$ and $\beta$ arethecomplex amplitudes of qubit states $|0\rangle$ and $|1\rangle$, and the superposition is resolved into either definite state $|0\rangle$ or $|1\rangle$ upon measurement with respective probabilities $|\alpha|^{2}$ and $|\beta|^{2}=1-|\alpha|^{2}$. In onesense, thisisjusta singlebit of information. But in another sense, thecontinuous amplitudes $\alpha$ and $\beta$ carry an infinite amount of information, similar to analogueinformation carrierssuch as the continuous voltage stored on capacitors. However, anal ogue systems are known to suffer from the cumulative build-up of noise, as opposed to digital standards liketransistor-transistor logic that latch to their high or low levels through constant measurement and feedback. Quantum bitsaresimilarly vul nerableto analoguenoise ${ }^{6}$, but they can offer much more in return: quantum entanglement. In a classical analogue system, we need $\mathrm{N}$ capacitors to store $\mathrm{N}$ continuous voltages. But with $\mathrm{N}$ qubits, the most general stateisspecified by $2^{\mathrm{N}}$ independent amplitudes $\gamma_{0}, \ldots, \gamma_{2} \mathrm{~N}-1$

$$
\begin{aligned}
|\Psi\rangle= & \gamma_{0}\left|0_{1} 0_{2} O_{3} \ldots 0_{N}\right\rangle+\gamma_{1}\left|0_{1} 0_{2} O_{3} \ldots 1_{N}\right\rangle+\ldots \\
& +\gamma_{2^{N}-1}\left|1_{1} I_{2} 1_{3} \ldots 1_{N}\right\rangle
\end{aligned}
$$

A collection of qubits therefore has the potential for storing exponentially moreinformation than a comparable collection of classical information carriers. (Of course, the trick is extracting this information following a measurement, as discussed in the below applications.) The above stateisentangled, as it is not generally separableinto a product of individual qubit states. The implicit interconnects from entanglement give a quantum information processor itspower (ref. 7; and seeBox 1).

Atoms and photons: good quantum hardware The chief hardware requirements for a quantum information processor are:

1. Thequantum system (that is, a collection of qubits) must beinitialized in a well-defined statesuch as $\left|0_{1} O_{2} O_{3} \ldots 0_{N}\right\rangle$.

2. Arbitrary unitary operators must be available and controlled to launch the initial state to an arbitrary entangled state( equation (2)).

3. M easurements of thequbitsmust beperformed with high quantum efficiency.

The first two requirements demand that the qubits are well isolated from the environment to ensure pure initial quantum states and to preserve their superposition character, but they must al so interact strongly between one another in order to become entangled. On the other hand, 

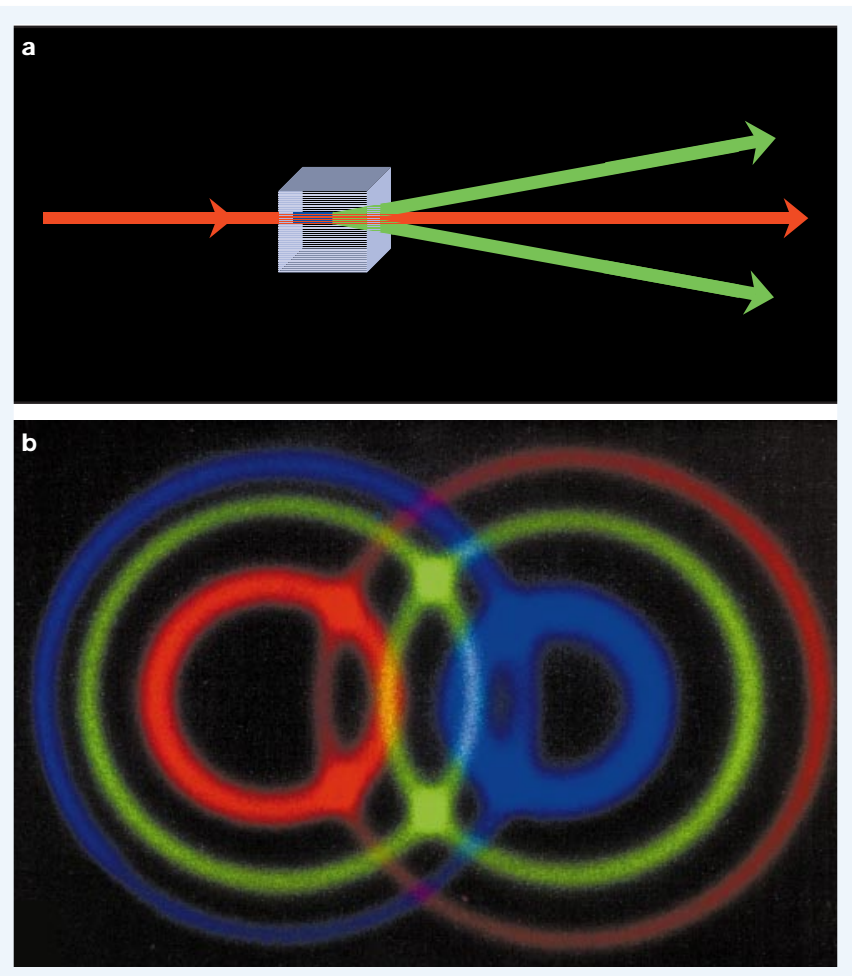

Figure 1 Optical parametric down-conversion. a, An ultraviolet photon incident on a nonlinear crystal can sometimes split spontaneously into two daughter photons. These photons are emitted on opposite sides of the pump beam, along two cones, one of which has horizontal polarization, the other of which has vertical polarization. b, Along the optical axis, several cone pairs can be seen. Photon pairs emitted along the intersections of the cones are entangled in polarization. (Image courtesy of $A$. Zeilinger, University of Vienna.)

the last stipulation requires the strongest possible interaction with theenvironment to beswitched on at will.

These stringent hardware requirements unfortunately rule out most known physical systems. Conventional solid-statearchitectures such assilicon are ideal for classical information for the samereason they are unsuitable for quantum information; as discussed above, their stability is given by the latching of logic levels, or continuous monitoring by the environment ${ }^{6}$. The most attractive candidates for quantum information processors currently come from the area of atomic physics and quantum optics ${ }^{9}$. Here, individual atoms and photons are manipulated in a controlled environment with wellunderstood couplings, offering environmental isolation that is unsurpassed in other physical systems.

Complex entangled states such as equation (2) can be generated by sequentially applying quantum logic-gate primitives to smaller numbers of qubits, similar to the use of sequences of universal logic gates such as NAND ('not-and') in classical computation. In fact, it has been shown that arbitrary entangled statescan begenerated from sequences of simplequbit logic gates acting on any one or two qubits at atime ${ }^{10,11}$. Thissequential model of quantum computing fitsnaturally with quantum optical and atomic systems, where interactions aregenerally weak and typically involveat most two qubits.

Quantum information processing requires qubits to behave as quantum memoriesfor long-term storageand for many applications to behave as quantum transmitters for long-distance communication. Cold and localized individual atoms are the natural choicefor qubit memories and sources of local entanglement for quantum information processing. The stability of quantum states in cold atomsisunrivalled, evidenced by thefact that such quantum systems currently host the world'sbest frequency standards(seereview in this issue by Udem, Holzwarth and $\mathrm{H}$ änsch, pages 233-237). Individual
Box 1

Entanglement, Schrödinger's cat and decoherence

Multiple quantum bits can be prepared in entangled states that are not able to be factored into a product of individual qubit states. An interesting class of entangled states is the 'Schrödinger-cat' states of $\mathrm{N}$ quantum bits, written as

$$
\left.\left.|\Psi\rangle_{\text {cat }}=\left|0_{1} 0_{2} 0_{3} \ldots 0_{N}\right\rangle+\left|1_{1} 1_{2} 1_{3} \ldots 1_{N}\right\rangle \approx||^{\prime} \text { live cat' }\right\rangle+\mid{ }^{\prime} \text { dead cat' }\right\rangle
$$

This state is known as a mesoscopic Schrödinger-cat state, as the constituents of the superposition are as far apart as possible, analogous to the live and dead feline. As Schrödinger himself noted, describing a macroscopic object such as a cat with quantum terms is of course ridiculous, but for mesoscopic systems where $\mathrm{N}$ is not too large, the above state is useful in grasping large-scale entanglement.

The qubits in a Schrödinger-cat state are perfectly correlated, yet each qubit is entirely random when considered alone. This hidden wiring of entanglement, responsible for gains in quantum information processing, can be roughly visualized in the following analogy ${ }^{89}$. Consider $\mathrm{N}=4$ adjacent three-dimensional cubes drawn with ambiguous perspective.

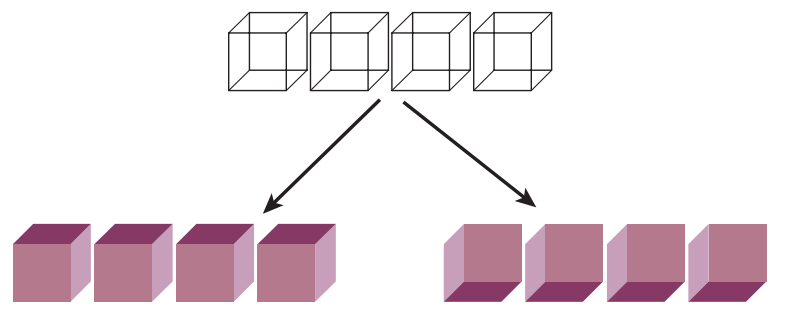

After a brief moment, your mind typically locks onto one of the two perspectives, and you will almost certainly see all cubes emerge with the same perspective. This is roughly analogous to a measurement of an entangled superposition.

The Schrödinger-cat state also highlights the difficulty in maintaining complex entangled quantum superpositions. If just one of the $\mathrm{N}$ qubits gets measured by the environment, every qubit loses its coherence. Decoherence makes it more difficult to engineer large entangled states, with the coherence survival probability decaying exponentially with the number of qubits ${ }^{90}$. Extrapolating to $\mathrm{N} \sim 10^{28}$ qubits in a real Schrödinger's cat, we find that the superposition of live and dead would almost instantaneously collapse into one or the other.

photons, on the other hand, are the natural sourcefor thecommunication of quantum information, as they can traverse large distances through theatmosphereor optical fibres with minimal disturbance.

\section{Quantum communication}

Imagine two distant parties, Alice and Bob, share two entangled qubitsanalogousto an Einstein-Podolsky-Rosen (EPR) state ${ }^{12}$

$\left|\Psi_{\mathrm{AB}}\right\rangle=\left|0_{\mathrm{A}} 0_{\mathrm{B}}\right\rangle+\left|1_{\mathrm{A}} 1_{\mathrm{B}}\right\rangle$

What can Aliceand Bob do with their entangled pair? At first glance, they might try to exploit the strong correlations in their entangled states for direct (and superluminal) communication. A measure ment by Alice or Bob immediately conveys the state of the other's qubit, but such measurements would yield only a sequence of perfectly random (yet correlated) bits, which carry no information according to Shannon. On theother hand, correl ated random strings 


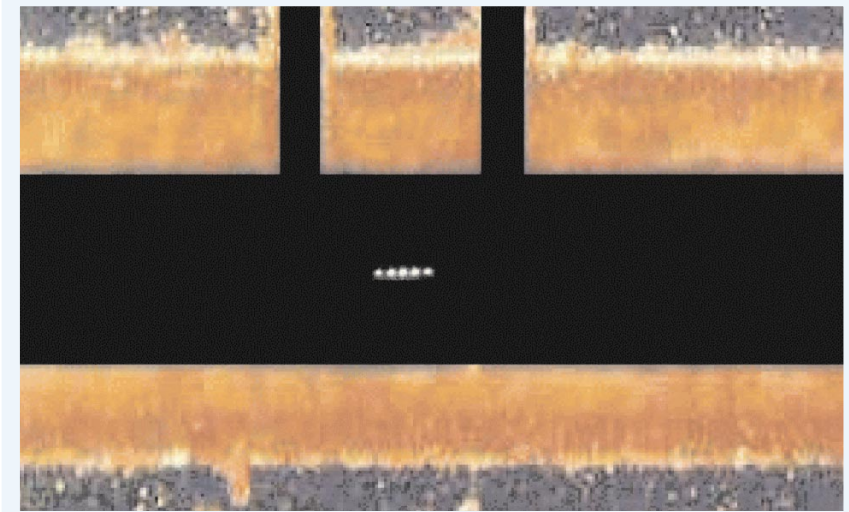

Figure 2 A crystal of five atomic beryllium ions (small white dots at centre) confined in a radio-frequency ion trap. The ions balance their mutual Coulomb repulsion with the confining force of electric fields generated from the surrounding electrodes (brown). The ions strongly fluoresce under the application of appropriate laser radiation near $313 \mathrm{~nm}$. The horizontal electrode gap is about $0.2 \mathrm{~mm}$ and the ion-ion spacing is $\sim 5 \mu \mathrm{m}$. (Image courtesy of NIST, Boulder.)

are extremely useful for cryptographic key distribution, as in the classic'one-timepad'13.

\section{Quantum cryptographic key distribution}

CharlesBennett and GillesBrassard developed the first protocolsfor quantum cryptographic key distribution (QKD) in 1984 (ref. 14), following earlier ideas of Steven Wiesner ${ }^{15}$. This scheme used the transmission of non-orthogonal states of single-photon qubits ${ }^{16}$, with security derived from the impossibility of an eavesdropper distinguishing the two states without being detected (on average). Several groups have implemented these protocols over tens of kilometres in underground fibres ${ }^{17-20}$. Instead of idealized singlephoton sources, these experiments used highly attenuated laser sources, which have a finiteprobability of emitting multiplephotons per pulse. This gives rise to a trade off between bit communication rate and security, resulting from the potential for eavesdropping on thesurplusphotons.

The use of entangled qubits can eliminate this trade-off and dramatically improve QKD. For example, by encoding qubits in orthogonal states of photon polarization in the form of equation (3), the measured presence of one photon at Alice indicates that Bob has exactly one photon, which can be used to improve the security of the abovesingle-qubit QKD scheme. Entangled qubits also offer an alternative QKD protocol, first pointed out by Artur Ekert in 1991 (ref. 21), and implemented recently by groupsat Vienna University ${ }^{22}$, Los Alamos National Laboratory ${ }^{23}$ and the University of Geneva ${ }^{24}$. Here, the security is derived from thefact that a potential eavesdropper disturbstheentanglement shared by Aliceand Bob.

Theseexperimentsused nonlinear optical parametric down-conversion (PDC) astheir sourceofEPR-likeentangled pairs. Ultraviolet pump photonsaredirected into anonlinear crystal that gives riseto a small probability of down-conversion into pairs of visibleor infrared daughter photons, as depicted in Fig. 1. These daughter photons alwaysappear simultaneously, and can beentangled in their polarization degrees of freedom with appropriate phase matching and mode selection. Unfortunately, PDC photon sources have several drawbacks in quantum information applications. Similar to the case of a highly attenuated laser source, there isa small probability of emitting pairs of down-converted photons that again offers an avenue for potential eavesdropping. Furthermore, PDC suffers from poor efficiency, as the probability of down-conversion per pump photon is typically below $10^{-10}$ ( ref. 25). The presence of an entangled pair can beinferred only after a measurement (post-selection), so such states cannot becascaded for subsequent processing to producelarge-scale entangled photon states. Nevertheless, PDC sources are very useful for demonstrating rudimentary quantum communication protocols such asQKD.

\section{Quantum teleportation}

How can qubits be transmitted between remote locations? One method is to simply move the qubits through space, similar to the transmission of classical bits with electrical currents. H owever, this can be very difficult in practice, especially over long distances, as it must be done without disturbing or measuring the qubit state. Quantum teleportation, discovered by Bennett in 1993 (ref. 26), offers an alternative method of transmission without physical con$\operatorname{tact}^{27}$, as described in Box 2. The drawback is that the sender and receiver must al ready possess entangled qubits, a situation that may be as difficult as physically moving qubits from $A$ to $B$ in the first place, as entanglement always originates between nearby qubits. The real power of quantum teleportation is that it allows quantum information to be conveyed over a channel that may be unsuitable for direct physical transmission at the time of communication, or a channel that cannot reliably host the form of qubit stored at each end. For example, teleportation might allow quantum states in
Figure 3 Schematic of an optical lattice. A pattern of crossed laser beams (right) creates an array of potential wells that can confine individual laser-cooled cold atoms (left). The atom- atom separation is of the order of the optical wavelength. (Image courtesy of S. Rolston, NIST.)

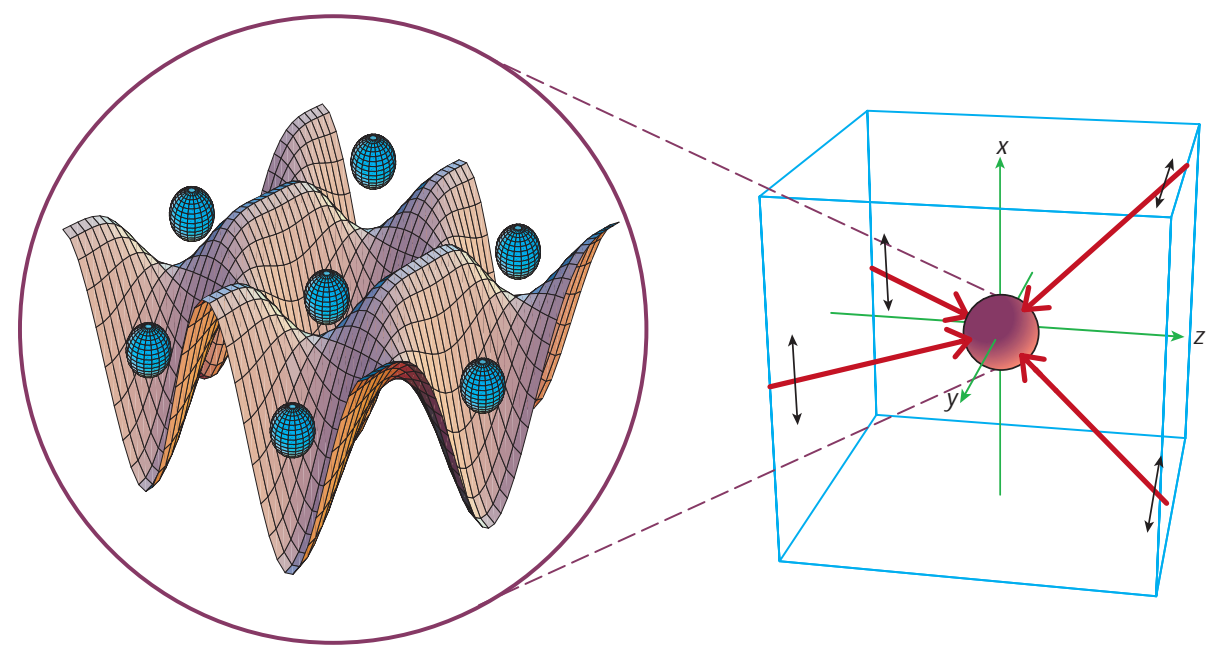


atoms to be communicated between remote locations that possess previously entangled photons.

Groups from the universities of Innsbruck ${ }^{28}$ and Rome $^{29}$ werethe first to demonstratecertain critical aspects of quantum teleportation in 1997. In these experiments, polarization qubits of single photons were teleported between spatially separated locations using PDC. The low efficiency of these sources precluded the teleportation of larger numbers of qubits or the recycling of qubits ${ }^{30,31}$. Shortly there after, a team at the California Institute of Technology (CalTech) efficientlyteleported thecontinuousquantum state of asingle-mode optical field ${ }^{32}$, relying on the deterministic entanglement of a pair of field modes, demonstrating anet fidelity of $58 \%$ without theneed for post-selection.

Will weever beableto teleport objects with morethan just asingle quantum degree of freedom? The answer is yes, as long as the three quantum hardware requirements are satisfied. But what about teleporting truly macroscopic objects with perhaps $10^{28}$ effective qubits? This prospect, about as likely as preparing a real Schrödinger's cat both alive and dead, is clearly "ridiculous" as Schrödinger himself said ${ }^{33}$. Instead, it is more fruitful to consider what we can do with a more modest system of perhaps 300 qubits, where the $\sim 10^{90}$ available quantum states are still more than the number of particlesin theUniverse.

\section{Quantum computing}

In 1985, David Deutsch showed how quantum superposition and entanglement could be harnessed to process information more efficiently than any classical machine $e^{34}$. Deutsch's 'quantum parallelism' allowsan $\mathrm{N}$-qubit quantum computer to operateon quantum superposition states of all $2^{\mathrm{N}}$ inputs. Extraction of information in a quantum computer is nontrivial, because simply measuring a quantum state such as equation (2) with an exponential number of nonzero amplitudes will yield a highly random result. In certain algorithms, however, appropriate quantum logic gates cause the amplitudes to interfere so that only a few amplitudes survive in the end. Following a measurement (or a limited number of repeated measurements on identical runs), the result (or distribution of results) can depend on aglobal property of all $2^{\mathrm{N}}$ inputs.

The best-known example of this procedure is Peter Shor's 1994 factoring al gorith $\mathrm{m}^{35}$, where a quantum computer would be able to factor large numbers exponentially faster than any known classical computer algorithm. Fast quantum number factoring has profound implications in cryptanalysis, as many popular encryption algorithmssuch as RSA (Rivest-Shamir-Adleman) ${ }^{36}$ rely on theinability to factor large numbers. In 1996, Lov Grover discovered a quantum al gorithm that would search an unsorted database of $M$ elementsin a time that scales roughly $\mathrm{M}^{1 / 2}$ faster than any possible classical search algorithm ${ }^{37}$. These discoveries have ushered a flurry of theoretical activity searching for further useful quantum computer applications.

A key development in 1995 was quantum error correction ${ }^{38,39}$, an extension of Shannon's famous 1948 noisy coding theorem ${ }^{1}$ to the quantum realm. Quantum error correction detects and corrects slight quantum gate errors or weak interactions with the environment through redundant encoding of qubits. This means that experimentsneed not beperfect; they requireonly that noiselevelsbebelow a certain threshold, with atrade-off between theamount of extra qubits required and the error threshold level. According to somemodel $\mathrm{s}^{40}$, arbitrary-length quantum computation can proceed error-freewith only a polynomial overhead in timeand space, so long as the error probability per fundamental operation is kept below about $10^{-5}$. What followsisa review of the most promising quantum computer architectures that may someday reach this level of fidelity.

Ion traps

Laser-cooled and trapped atomic ions represent one of the most attractive candidates for a large-scal e quantum computer ${ }^{41-44}$. Here, electromagnetic fields confine individual atoms in free space in a
Box 2

Quantum teleportation

Quantum teleportation, the 'disembodied transport' of a quantum state from one site to another ${ }^{91}$, is conceptually simple, as outlined in the figure below. Imagine that Alice and Bob each already possess one of two qubits prepared in an entangled state such as equation (3). If Alice prepares another qubit $A^{\prime}$ in the arbitrary state $\alpha|0\rangle_{\mathrm{A}^{\prime}}+\beta|1\rangle_{\mathrm{A}^{\prime}}$, the overall state of the three qubits is

$$
\begin{aligned}
|\Psi\rangle & =\left(\alpha|0\rangle_{A^{\prime}}+\beta|1\rangle_{A^{\prime}}\right)\left(\left|0_{A}\right\rangle\left|0_{B}\right\rangle+\left|1_{A}\right\rangle\left|1_{B}\right\rangle\right) \\
& =\left|\phi^{+}\right\rangle_{A^{\prime} A}\left(\alpha|0\rangle_{B}+\beta|1\rangle_{B}\right)+\left|\phi^{-}\right\rangle_{A^{\prime} A}\left(\alpha|0\rangle_{B}-\beta|1\rangle_{B}\right) \\
& +\left|\psi^{+}\right\rangle_{A^{\prime} A}\left(\alpha|1\rangle_{B}+\beta|0\rangle_{B}\right)+\left|\psi^{-}\right\rangle_{A^{\prime} A}\left(\alpha|1\rangle_{B}-\beta|0\rangle_{B}\right)
\end{aligned}
$$

where the four 'Bell' states of the Alice's qubits are defined as

$\left|\phi^{ \pm}\right\rangle \equiv\left|0_{A^{\prime}}, 0_{A}\right\rangle \pm\left|1_{A^{\prime}} 1_{A}\right\rangle$

$\left|\psi^{ \pm}\right\rangle \equiv\left|0_{A^{\prime}} 1_{A}\right\rangle \pm\left|1_{A^{\prime}} 0_{A}\right\rangle$

To pass the quantum information of Alice's qubit (the amplitudes $\alpha$ and $\beta$ ) to Bob, Alice makes a perfect projection measurement of her qubit pair onto this complete basis of Bell states. This can be done with simple operations of quantum logic gates on qubits $A$ and $A^{\prime}$ along with an efficient measurement of both qubits, as depicted in the figure. This measurement immediately projects Bob's lone qubit onto one of the four states correlated with the state measured by Alice given in equation (5), resulting in his qubit now carrying the quantum information $\alpha$ and $\beta$. After Alice communicates which Bell state she measured to Bob using classical means (for example, a phone call), Bob performs a prescribed local qubit manipulation on his one qubit to replicate the initial state $\alpha|0\rangle+\beta|1\rangle$ in his qubit. For example, if Alice reports a measurement of $\left|\phi^{+}\right\rangle$, then Bob does nothing to his qubit (operation I). If Alice instead reports a measurement of $\left|\psi^{-}\right\rangle$, then Bob flips his qubit (operation $\mathrm{F}$ ) and additionally adds a $\pi$-phase shift (operation $\mathrm{P}$ ). In this way, the qubit $\alpha|0\rangle+\beta|1\rangle$ is 'teleported' from Alice to Bob without disturbing or measuring the qubit.

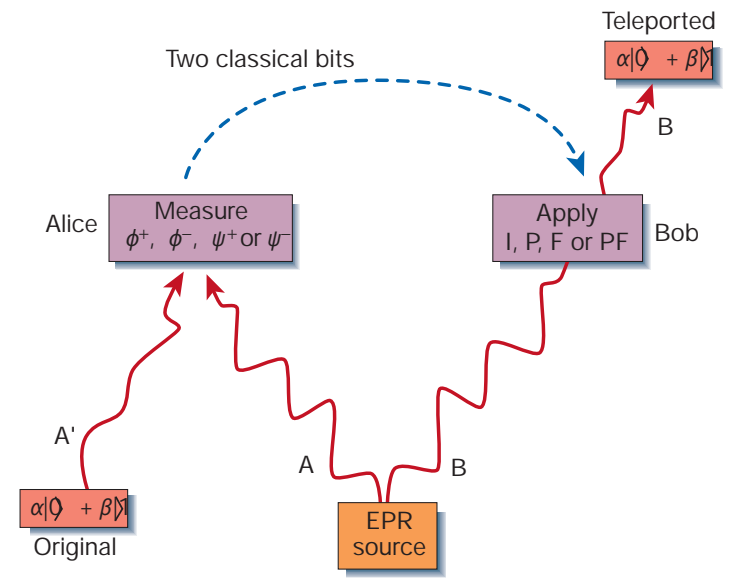

vacuum chamber, and when multiple ions are confined and lasercooled, they form simple stationary crystal structures given by the balance of the external confining force of the trap with the mutual repulsion of theatoms (Fig. 2). Qubitsarestored in internal electronic states of the atoms, typically the same long-lived hyperfine states that are used in atomic clocks. When appropriate laser radiation is directed to the atomic ions, qubit states can be mapped coherently onto the quantum state of collective motion of the atoms and subsequently mapped to other atoms. A singlenormal mode of collective 


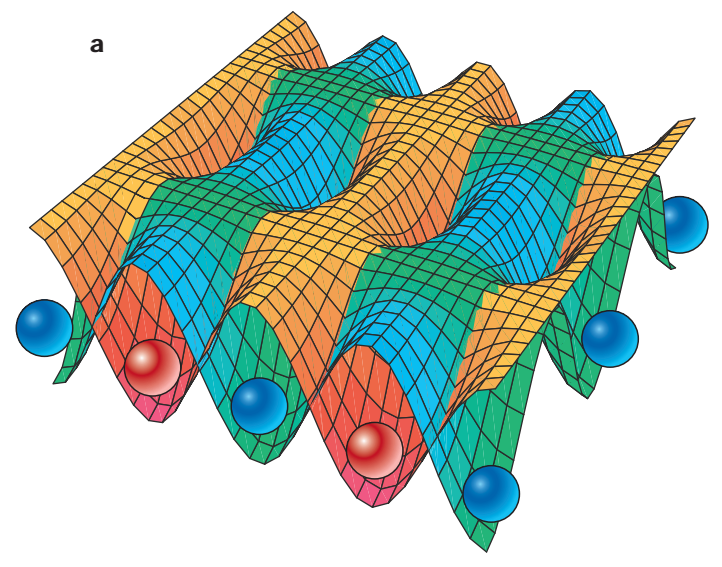

Figure 4 Optical lattice potentials. a, Optical lattice potentials can depend on the internal state (red or blue) of the confined atom qubits. $\mathbf{b}$, These complementary lattice potentials can be controlled by varying the polarization of one of the lattice light fields,

crystal motion thus behaves as a quantum data-bus, allowing quantum information to beshared and entangled between remoteatomic qubits in the crystal. Finally, theinternal states of individual trapped ions can be measured with nearly $100 \%$ quantum efficiency ${ }^{45}$ by applying appropriate laser radiation and collecting fluorescence onequbit stateisbright and theother isdark.

Quantum logic gates have been demonstrated with up to four trapped atomicion qubits at theNational Institute of Standards and Technology (NIST) at Boulder, Colorado ${ }^{46,47}$. Although this scheme is in principle scalable to arbitrarily large numbers of qubits, the main problems deal with the collective motion of the atoms. The quantum gate speed is limited to the frequency of motion, typically in thesub-megahertz range. As morequbits are added to the collection, the density of motional states balloons, and isolation of asingle mode of motion (for example, the centre-of-mass) becomes even moreslow and difficult. M oreover, external noisy el ectric fieldstend

Figure 5 Scheme for a scalable ion trap and optical-lattice quantum computer. An array of independent (memory) ion traps is coupled through interaction with a roving (head) ion trap. A strong optical-lattice laser field entangles the head ion with a given memory or target ion. (Image courtesy of P. Zoller, University of Innsbruck.)

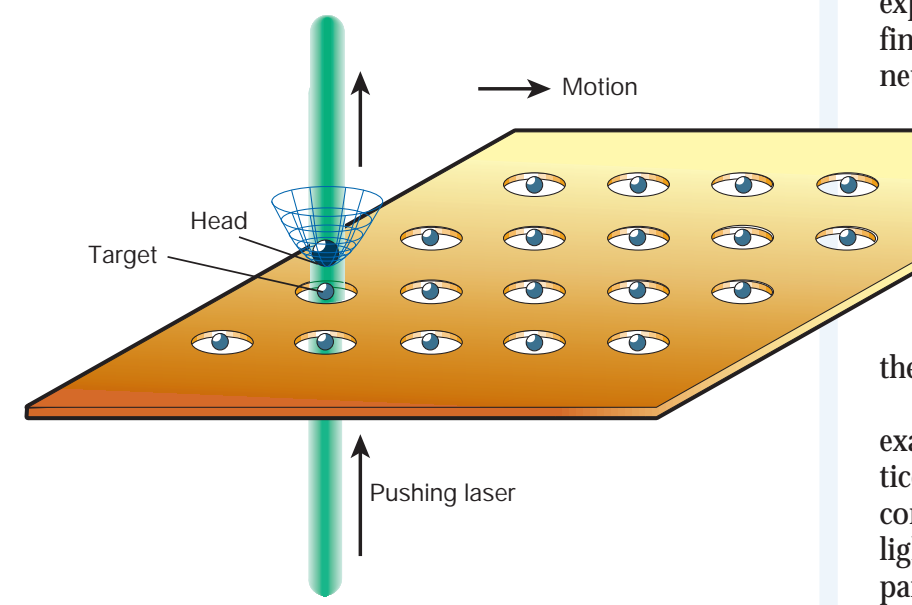

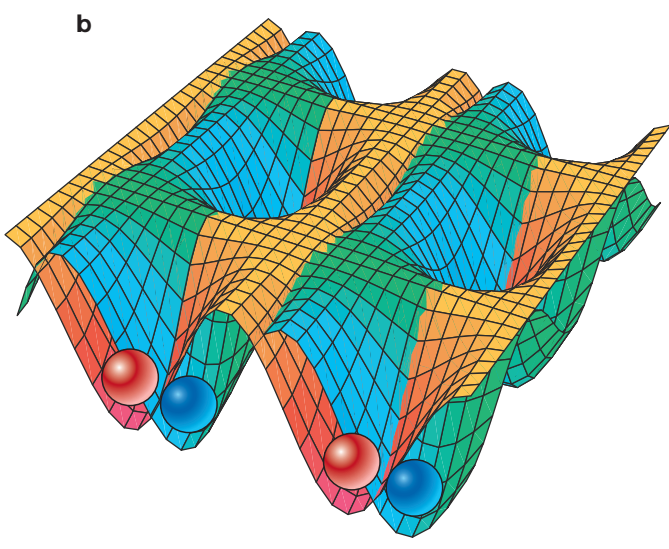

shifting the interference pattern and bringing atoms together in pairs for quantum logic gates. (Image courtesy of I. Deutsch, University of New Mexico.)

to compromise the motional coherence of large numbers of trapped atomicions ${ }^{48}$.

A promisingapproach that attacksboth problemsis thequantum charged-coupled device (CCD), where individual atomic ions are entangled as above, but only among a small collection (under 10) of atomic ions in an 'accumulator' 43 . Scaling to larger numbers in accomplished by physically swapping individual atoms between the accumulator and a 'memory' reservoir of trapped atom qubits. This can bedonequickly with externally applied electricfieldsin elaborate ion-trap electrodegeometries. Thekey features of thequantum CCD arethat theion shuttling can bedonewithout perturbing theinternal qubits, and the motional quantum state of the ions factors from the internal qubit states following quantum gate operation. In order to quench the motional energy from rapid shuttling and allow subsequent logic gates, ancillary ions in the accumulator can be lasercooled between gate operations. The qubit ions are thereby sympathetically cooled ${ }^{49}$ through their strong Coulomb interaction with these extra refrigerator ions. Sympathetic cooling can also eliminate motional decoherenceduring logic gateoperation ${ }^{43,50,51}$.

Cold atoms and dipoles in optical lattices

Neutral atom qubits enjoy a weak coupling to theenvironment, at the expense of a weak dipole-dipole coupling between each other. To exploit this coupling for entanglement, atoms must be tightly confined and controlled to sub-micron dimensions. A natural host of neutral atomsfor quantum information purposesistheoptical lattice - an array of cold atoms confined in free space by a pattern of crossed laser beams $s^{52}$. The dipole force between the polarizable atoms and the field results in a regular pattern of potential wells, thespacing of which is of theorder of an optical wavelength (Fig. 3). Several groups havelaser-cooled atomsto the lowest bound statein latticewells $s^{53-55}$ and controlled their localized quantum wavepacket states ${ }^{56,57}$.

Optical lattice potentials generally depend upon qubit level (for example, one state's valley can be another state's hill). Atoms in lattices can therefore be shifted to nearly overlap with their neighbours, conditioned upon their internal qubit state, by modulating thelattice light polarization or intensity, as shown in Fig. 4. One proposal ${ }^{58}$ pairs adjacent atoms together with one of the atoms prepared in a short-lived excited state. The resultant resonant dipole-dipole interaction, mediated by the shared excitation, allows pair-wise 
entanglement. Thespeed of thisquantum gatemust befaster than the excited-state decay time, meaning the atoms must be localized and overlapped to well under an optical wavelength. A related proposal would transiently bring adjacent atoms together depending on their internal qubit levels, but instead rely on 'cold collisions' for entangling logic gates ${ }^{59}$. If theatoms aresufficiently cold, then such s-wave collisions will result in a conditional phase shift of their quantum states, or a quantum phasegate.

Another approach exploits the large electric dipole moments of Rydberg atoms ${ }^{60}$ or even simple heteronuclear molecules ${ }^{61}$ in an externally applied electric field. For instance, selected atoms held in an optical lattice can be entangled by promoting them to Rydberg states and relying on their mutual dipole-dipole interaction. The interaction strengths over micrometre distances can be in the gigahertz regime, implying very fast quantum gateoperation.

Onesignificant drawback to the use of optical latticesin quantum information is that the lattice sites are typically not uniformly packed. H owever, the samestatistical properties of bosons responsiblefor Bose-Einstein condensation (seereview in thisissueby Anglin and Ketterle, pages 211-218) can be exploited for better control of atom number in optical lattices. A subtle interplay between a repulsive s-wave scattering interaction between bosonic atoms and the tunnelling of atoms between lattice sites can smooth out the atom-number statistics between wells, a phenomenon known as a Mott-insulator phase transition ${ }^{62,63}$. Signatures of this behaviour have been observed in an optical latticeloaded with a Bose-Einstein condensate. Researchers at Yale University ${ }^{64}$ measured fluctuations in the number $\mathrm{N}$ of atoms per lattice site by coherently combining atoms in adjacent sites in an atom interferometer, and reported a spread in atom number that is afactor of about 40 below thestandard $\mathrm{N}^{1 / 2}$ shot-noise level. A group at the M ax Planck Institute for Quantum Optics (MPQ) in Garching, near M unich, has recently observed signatures of the small $\mathrm{N}$ limit to such 'number-squeezed' states, where exactly one atom resides in each site $e^{65}$. This sets the stage for futureprogressin quantum gateoperationsusing optical lattices.

A further proposal combines the features of optical lattices and ion traps, where individual ions are entangled through a common interaction with a pulsed, high-strength optical lattice ${ }^{66}$. The lattice jerks two ions such that the internal qubit states of each ion are mapped to displaced spatial positions, smearing out their chargedistributions ${ }^{67}$. Theinteraction between these'engineered' dipolesthen allows entangling quantum logic gates that can be much faster than theion-trap frequency. M oreover, purequantum statesof motion are not necessary, so long as the ions are confined to much less than the ion-ion separation. Finally, the ions need not be close together or even in the same trap, providing a convenient method for scaling to arbitrary numbers of qubitsin an array of separateion traps(Fig. 5).

\section{Quantum networks}

As more complex quantum communication protocols are demonstrated, therewill bea pressing need to storequantum information in long-term memories. To construct reliable quantum networks of both quantum transmittersand quantum memories, small quantum computers will be needed at the nodes, acting as routers, repeaters and switches.

Degradation of quantum information will be unavoidable when qubits are sent over large distances or over noisy channels. This will requirequantum 'repeaters' to beplaced periodicallyalong thechannel so that qubit errorscan becor rected. These repeaters can takethe form of error-correcting quantum computers, or stations that share several imperfect entangled qubit pairs with adjacent sites ${ }^{68}$. Here, quantum computer operations at the nodes 'purify' the entangled states so that states can effectively be teleported over remote distances. Remarkably, some of these protocols require local operation fidelities of only $98 \%$ for successful transmission ${ }^{69}$.

A key component of such a quantum network is the faithful and coherent conversion of quantum information between different

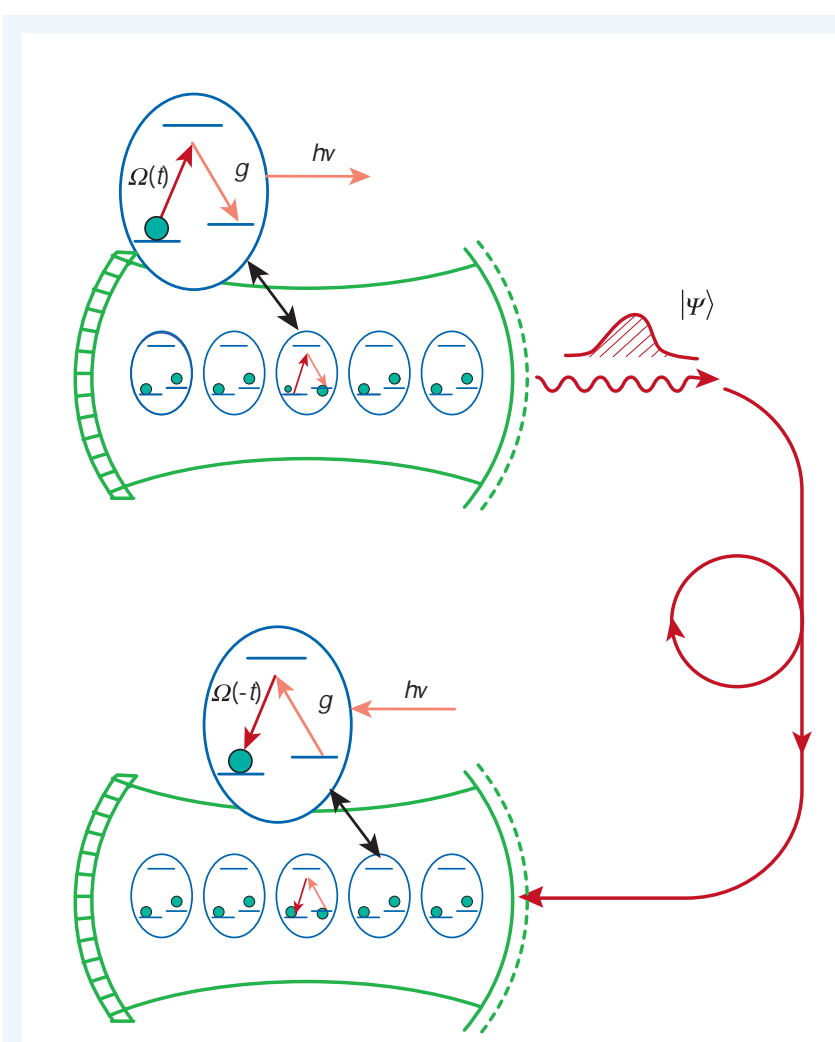

Figure 6 Atom- photon quantum network. A selected atom inside the top optical cavity coherently transfers its internal qubit onto a single-photon qubit in the cavity through the application of a classical laser pulse represented by the coupling $\Omega(t)$. The coupling $g$ is between the single-photon field in the cavity and the atom. The single photon leaks out of the top cavity, only to be caught in the lower cavity by a time-reversed and synchronized classical laser pulse $\Omega(-t)$. (Image courtesy of H. J . Kimble, CalTech.)

systems. Of particular interest is the reversible mapping of qubits from photon statesto atomic states. This might al low theimplementation of photonic quantum repeaters and also the teleportation of qubits from remotely located atoms through common EPR pairs of photons.

Cavity quantum electrodynamics and cold atoms

An elegant proposal to marry atomic and photonic quantum bits in optical cavity quantum electrodynamics (QED) was developed in 1997 (ref. 70). In this scheme, a small number of cold atoms areconfined to the antinodes of a single-photon standing-wave field in a high-finesse Fabry-Perot optical cavity. Qubit states stored in the internal states of the atoms can be mapped to the qubit spanned by thenumber of photons( 0 or 1 ) in thecavity through application of an appropriatelaser pulsefrom theside. Thephoton can bemadeto leak out of the cavity within a pre-set time window, resulting in an ideal singlephoton source for use in quantum communication ${ }^{71}$. M oreover, after thephoton leaksout of thecavity, it can bedeterministically 'caught' in a second cavity by the application of another laser pulse, whoseenvelopeistimereversed with respect to thefirst pulse, as depicted in Fig. 6. Generalizations involving more than one atom in each cavity can distributeentanglement to many nodes while also featuring fault-tolerant error correction ${ }^{72}$.

Applying cavity-QED techniques to quantum information requires a single-atom/single-photon coupling that overwhelms any loss or decoherencerate, including atomic spontaneous emission and photon leakage through the mirrors. This leads to cavities of small 
Figure 7 Single-atom cavityQED experiment. Cold caesium atoms are dropped into a high-finesse optical cavity of axial spacing $\sim 10 \mu \mathrm{m}$. The trajectory of a single atom traversing the cavity is reconstructed (inset) by monitoring the field that leaks out of the cavity. (Image courtesy of H. J. Kimble, CalTech)

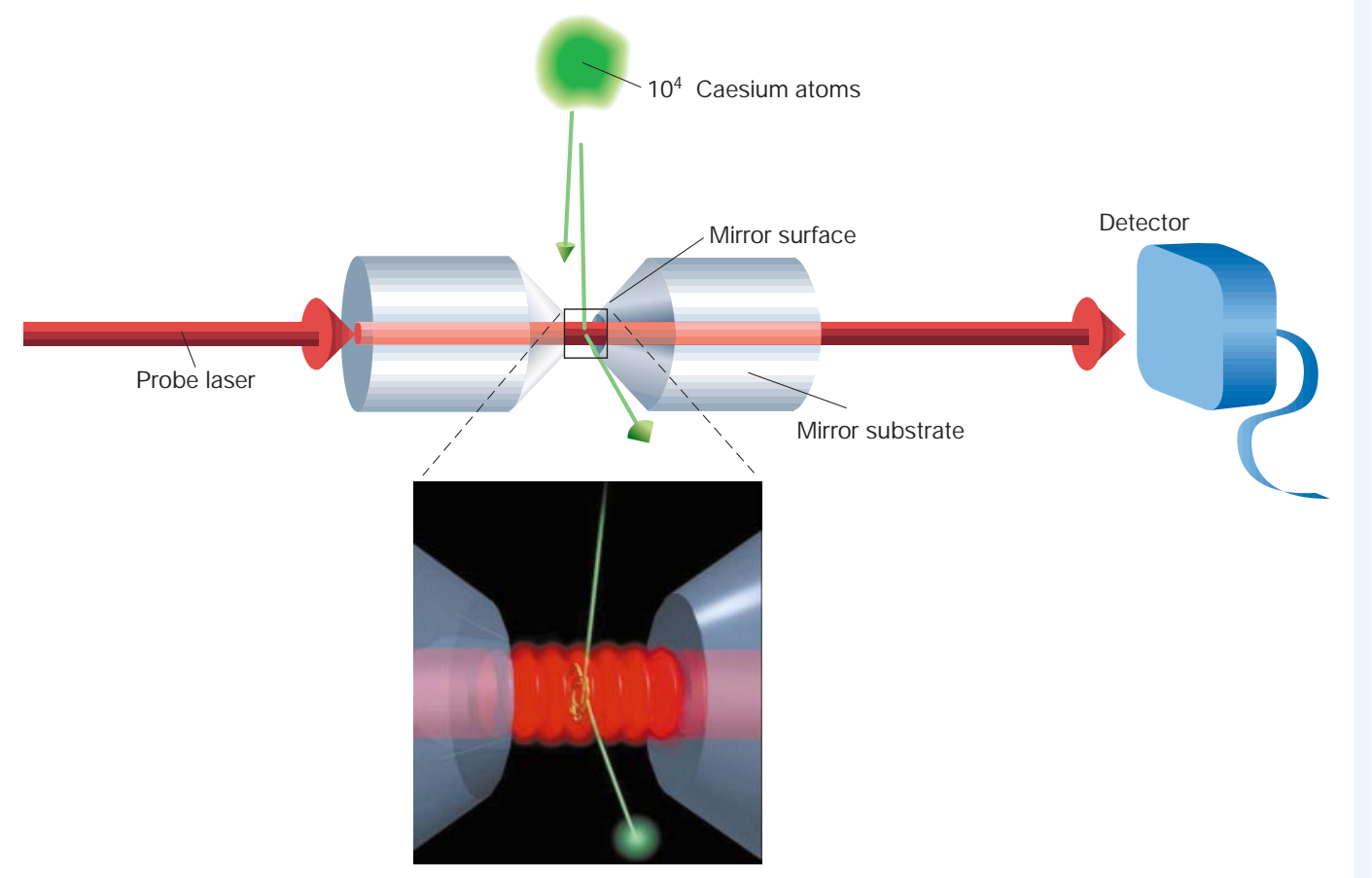

volume(for a high single-photon intensity) and extremely high mirror reflectivity (to keep thephoton in thecavity). Groups from CalTech ${ }^{73,74}$ and $\mathrm{MPQ}^{75}$ are beginning to master this difficult technology by trapping individually laser-cooled atoms inside miniature high-finesse cavities (Fig. 7). The atoms evolve in the requisite 'strong-coupling' limit, where even single photons in the cavity can significantly influence the dynamics of atomic motion ${ }^{76}$. However, the atomic trajectories are still extended when compared to the optical wavelength, which effectively randomizes the atom-photon coupling and leads to decoherence. A second M PQ group is progressing on a complementary system, where individual atomic ions have been tightly bound within alarger optical cavity so that theatomic trajectory iswell under an optical wavelength ${ }^{77}$. But here the atom-photon system is not strongly coupled, because the insulating surfaces of a smaller cavity could interferewith theion-trapping fields. Theseexperiments, along with approaches from several other groups, point theway towards an ultimategoal in quantum information networking - that of coherently exchangingsingleatomsand singlephotons.

\section{'Stopping' light}

Several recent experiments from the universities of $\mathrm{H}$ arvard ${ }^{78,79}$ and Berkeley ${ }^{80}$ have reported slowing and even 'stopping' light using the techniques of electromagnetically induced transparency ${ }^{81}$ in an atomic vapour. Thestopping of light might seem trivial at first glance - it can beeasi ly accomplished by putting an opaqueblack screen in front of a light source. Instead of irreversibly destroying thephotons, however, theseexperimentsoffer thepotential for mappingquantum states of photons onto collective quantum states of the atomic vapour $^{82}$. For example, single-photon qubits can be mapped onto a singlequbit spanningall $N$ spinsin avapour:

$\left[\alpha|0\rangle_{\gamma}+\beta|1\rangle_{\gamma}\right]\left|0_{\text {exc }}\right\rangle \rightarrow|0\rangle_{\gamma}\left[\alpha\left|0_{\text {exc }}\right\rangle+\beta\left|1_{\text {exc }}\right\rangle\right]$

where $|1\rangle_{\gamma}$ and $|0\rangle_{\gamma}$ represent the presence or absence of the photon, respectively, and $\left|0_{\text {exc }}\right\rangle=\left|\downarrow_{1} \downarrow_{2} \downarrow_{3} \ldots \downarrow_{N}\right\rangle$ and $\left|1_{\text {exc }}\right\rangle=\left[\left|\uparrow_{1} \downarrow_{2} \downarrow_{3} \ldots \downarrow_{N}\right\rangle+\right.$ $\left.\left|\downarrow_{1} \uparrow_{2} \downarrow_{3} \ldots \downarrow_{N}\right\rangle+\ldots+\left|\downarrow_{1} \downarrow_{2} \downarrow_{3} \ldots \uparrow_{N}\right\rangle\right] / N^{1 / 2}$ are the symmetric states of zero and one excitation, respectively. Recent work from Harvard has demonstrated classical coherence in this process ${ }^{83}$, although quantum coherenceinvolving small numbers of photons has not yet been observed.

A drawback in this mapping is the requirement of quantum number states of photons in the first place, which may ultimately require the cold atom and cavity-QED systems mentioned above. Oneway around this requirement is to exploit interactions between theatoms in the vapour to generate quantum states of excitation. If a classical laser pulseexcites atoms to Rydberg states for instance, then a strong resonant dipole-dipole interaction can inhibit (shift spectroscopically) further excitations, leaving the state $\left|1_{\text {exc }}\right\rangle$, or exactly oneexcited Rydberg atom in thecollection, a phenomenon called the 'dipoleblockade'84.

An alternative quantum network might map the continuous degrees of freedom of a light field (for example, polarization or electric-field quadratures) onto a collection of atoms. In this way, atoms can be entangled in symmetric ('spin-squeezed') states with many excitations, an extension of states like $\left|1_{\text {exc }}\right\rangle$ above $e^{85,86}$. A group at the University of Aarhus has recently entangled spatially separated collections of atoms through common interaction with a light field that traverses both collections (ref. 87; and see Fig. 8). In this case, a macroscopic number of atomsismadeto behaveas a singlequantum degree of freedom, and such a system has promise for teleporting states between remotely located quantum memories ${ }^{88}$. These experiments can be remarkably simple, requiring only trivial optical equipment and thermal samples of atomsin vapour cells.

\section{Conclusions}

Quantum information technology is likely to havean important role in information processing after the demise of Moore's Law. The extent of this role is unknown, as quantum information processors currently haveonly a limited number of known applications, such as number factoring, database searches and enhanced communication 


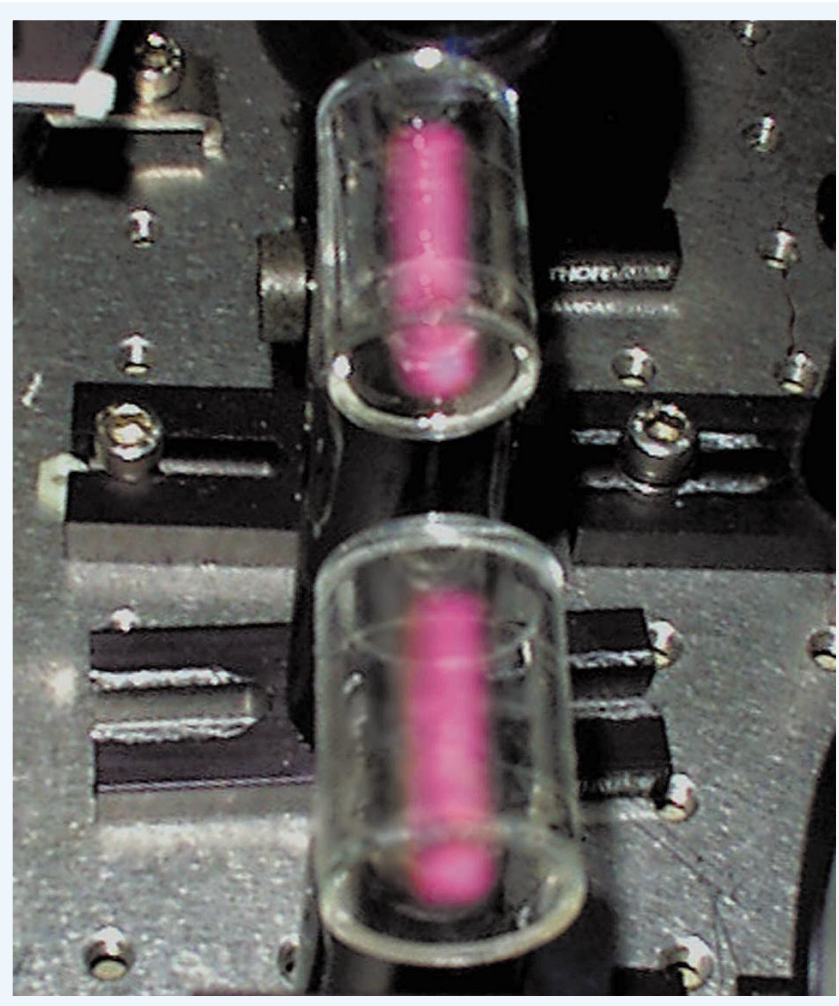

Figure 8 Pair of rubidium vapour cells used for storage and entanglement. A common laser beam traverses both cells, resulting in the purple fluorescence glow. Particular measurements of the polarization of the output light projects the atom collections in a 'spin-squeezed' state where the collective magnetization of the two macroscopic samples is weakly entangled. (Image courtesy of E. Polzik, University of Aarhus.)

protocols. M oreover, it may take decadesto learn how to build largescale quantum hardware, as this revolutionary form of information processing demands that revolutionary and exotic devices be engineered. Current devices come primarily from the areas of quantum optics and atomic physics, usually involving laser-cooled and trapped atoms. But perhaps the most exciting feature of this field is that the first large-scale quantum computer will probably be built from aphysical system that isnot currently known.

To quote Richard Feynman: "I think it is safe to say that nobody understands quantum mechanics." Current experiments that control individual atoms and photons will continue to lead the bizarre features of quantum-mechanical foundations to the fore front. After all, the systems currently under study are exactly the thought experiments envisioned by Einstein, Bohr and the other founding fathers of quantum physics. With the new language of quantum information, we might hope to gain more insight in the underlying quantum-physical principles, exactly as Shannon's theory of classical information ushered advances in physics responsible for thecurrent digital age.

1. Shannon, C. E. A mathematical theory of communication. Bell Syst. Tech. J. 27, 379-423; 623-656 (1948).

2. Benioff, P. The computer as a physical system: a microscopic quantum mechanical model of computers as represented by Turing machines. J. Stat. Phys. 22, 563-591 (1980).

3. Benioff, P. Quantum mechanical Hamiltonian models of Turing machines that dissipateno energy. Phys. Rev. Lett. 48, 1581-1585 (1982)

4. Feynman, R. Simulating physics with computers. J. Theor. Phys. 21, 467-488 (1982)

5. Feynman, R. P. There's plenty of room at the bottom. Eng. Sci. February 1960 issue (produced by the CalTech Office of Public Relations) (1960); also availableat <http://www.zyvex.com/nanotech/ feynman.html>.

6. Landauer, R. in Proc. D rexel-4 Symp. Quantum Nonintegrability (edsFeng, D. H. \& Hu, B.-L.) 44-53 (International Press, Boston, 1997).

7. Jozsa, R. in The Geometric Universe(ed. Huggett, S. et al.) 369-379 (Oxford Univ. Press, Oxford, 1998); preprint quant-ph/9707034 (Entanglement and quantum computation) at http://xxx.Ianl.gov (1997)

8. DiVincenzo, D. The physical implementation of quantum computation. Fortschr. Phys. 48, 771-783 (2000)
9. Special issue on experimental proposals for quantum computation. Fortschr. Phys. 48 (2000)

10. DiVincenzo, D. Two-bit gates are universal for quantum computation. Phys. Rev. A 51, 1015-1022 (1995)

11. Lloyd, S. Almost any quantum logic gate is universal. Phys. Rev. Lett. 75, 346-349 (1995).

12. Einstein, A. Podolsky, B. \& Rosen, N. Can quantum-mechanical description of reality be considered complete? Phys. Rev. 47, 777-780 (1935)

13. Kahn, D. The Codebreakers: TheStory of Secret Writing (M acmillan, New York, 1967).

14. Bennett, C. \& Brassard, G. Q uantum cryptography: public key distribution and coin tossing. Proc. IEEE Int. Conf. Comp. Syst. Signal Proc. 11, 175-179 (1984).

15. Wiesner, S. Conjugate coding. Sigact N ews 15, 78-88 (1983).

16. Bennett, C. Quantum cryptography using any two nonorthogonal states. Phys. Rev. Lett. 68, 3121-2124 (1992).

17. Franson, J. \& Ilves, H. Q uantum cryptography using optical fibers. Appl. O pt. 33, 2949-2954 (1994).

18. M arand, C. \& Townsend, P. Quantum key distribution over distances as long as $30 \mathrm{~km}$. O pt. Lett. 20, $1695-1697$ (1995).

19. Muller, A., Zbinden, H . \& Gisin, N. Quantum cryptography over $23 \mathrm{~km}$ in installed under-lake telecom fiber. Europhys. Lett. 33, 335-339 (1996).

20. Hughes, R. J., M organ, G. L. \& Peterson, C. G. Quantum key distribution over a 48km optical fibre network. J. M od. O pt. 47, 533-547 (2000).

21. Ekert, A. K. Quantum cryptography based on Bell's theorem. Phys. Rev. Lett. 67, 661-663 (1991)

22.Jennewein, T., Simon, C., Weihs, G., Weinfurter, H . \& Zeilinger, A. Quantum cryptography with entangled photons. Phys. Rev. Lett. 84, 4729-4732 (2000).

23. Naik, D. S., Peterson, C. G., White, A. G., Berglund, A. J. \& Kwiat, P. G. Entangled statequantum cryptography: eavesdropping on theEkert protocol. Phys. Rev. Lett. 84, 4733-4736 (2000)

24. Tittel, W., Brendel, J., Zbinden, H . \& Gisin, N. Quantum cryptography using entangled photons in energy-timeBell states. Phys. Rev. Lett. 84, 4737-4740 (2000)

25. Kwiat, P. G., Waks, E., White, A. G., Appelbaum, I. \& Eberhard, P. H. Ultrabright source of polarization-entangled photons. Phys. Rev. A 60, 773-776 (1999).

26. Bennett, C. H. et al. Teleporting an unknown quantum state via dual classical and Einstein-PodolskyRosen channels. Phys. Rev. Lett. 70, 1895-1899 (1993)

27. Braunstein, S. L. A fun talk on teleportation. <www.informatics.bangor.ac.uk/ schmuel/tport.html> (5February 1995).

28. Bouwmeester, D. et al. Experimental quantum teleportation. Nature 390, 575-579 (1997).

29. Boschi, D., Branca, S., DeM artini, F., H ardy, L. \& Popescu, S. Experimental realization of teleporting an unknown pure quantum statevia dual classical and Einstein-Podolsky-Rosen channels. Phys. Rev. Lett. 80, 1121-1125 (1998).

30. Braunstein, S. L. \& Kimble, H. J. A posteriori teleportation. Nature 394, 840-841 (1998).

31. Bouwmeester, D. et al. Reply to 'A posteriori teleportation' by S. L. Braunstein and H. J. Kimble. Nature 394, 841 (1998).

32. Furusawa, A. et al. Unconditional quantum teleportation. Science 282, 706-709 (1998).

33. Schrödinger, $E$. Thepresent situation in quantum mechanics. Naturwissenschaften 23, 807-812; 823-828; 844-849 (1935).

34. Deutsch, D. Quantum theory, the Church-Turing principle and the universal quantum computer. Proc. R. Soc. Lond. A 400, 97-117 (1985).

35. Shor, P. Polynomial-timealgorithms for primefactorization and discrete logarithms on a quantum computer. SIAM J. Comp. 26, 1484-1509 (1997).

36. Rivest, R., Shamir, A.\& Adleman, L. A method for obtaining digital signatures and public-key cryptosystems. Commun. Assoc. Comput. M ach. 21, 120-126 (1978).

37. Grover, L. Quantum mechanics helps in searching for a needlein a haystack. Phys. Rev. Lett. 79, 325-328 (1997).

38. Shor, P. Schemefor reducing decoherence in quantum computer memory. Phys. Rev. A 52, 2493-2496 (1995).

39. Steane, A. Error correcting codes in quantum theory. Phys. Rev. Lett. 77, 793-797 (1996)

40. Preskill, J. Battling decoherence: the fault-tolerant quantum computer. Phys. Today 52, $24-30$ (1999)

41. Cirac, I. \& Zoller, P. Quantum computations with cold trapped ions. Phys. Rev. Lett. 74, 4091-4094 (1995).

42. Steane, A. Theion trap quantum information processor. Appl. Phys. B 64, 623-642 (1997).

43. Wineland, D. et al. Experimental issues in coherent quantum manipulation of trapped atomic ions. J. Res. Natl Inst. Stand. Tech. 103, 259-328 (1998).

44. Sørensen, A. \& M ølmer, K. Quantum computation with ions in thermal motion. Phys. Rev. Lett. 82, 1971-1975 (1999).

45. Blatt, R. \& Zoller, P. Quantum jumps in atomic systems. Eur. J. Phys. 9, 250 (1988)

46. M onroe, C., M eekhof, D. M., King, B. E., Itano, W. M . \& Wineland, D. J. Demonstration of a universa quantum logic gate. Phys. Rev. Lett. 75, 4714-4717 (1995).

47. Sackett, C. et al. Experimental entanglement of four particles. Nature 404, 256-259 (2000).

48. Turchette, Q. A. et al. Heating of trapped ions from the quantum ground state. Phys. Rev. A 61, 063418-1-063418-8(2000).

49. Larson, D. J. et al. Sympathetic cooling of trapped ions: a laser-cooled two-species nonneutral ion plasma. Phys. Rev. Lett 57, 70 (1986).

50. Kielpinski, D. et al. Sympathetic cooling of trapped ions for quantum logic. Phys. Rev. A 61, 0323101-032310-8(2000).

51. M origi, G. \& Walther, H. Two-species Coulomb chains for quantum information. Eur. Phys. J. D 13, 261-269 (2001)

52.Jessen, P. \& Deutsch, I. O ptical lattices. Adv. At. M ol. O pt. Phys. 37, 95-138 (1996).

53. Hamann, S. et al. Resolved-sideband Raman cooling to the ground state of an optical lattice. Phys. Rev. Lett. 80, 4149-4152 (1998).

54. Vuletic, V., Chin, C., Kerman, A. J. \& Chu, S. Raman sideband cooling of trapped cesium atoms at very high densities. Phys. Rev. Lett. 81, 5768-5771 (1998)

55. Perrin, H., Kuhn, A., Bouchoule, I. \& Salomon, C. Sideband cooling of neutral atoms in a far-detuned optical lattice. Europhys. Lett. 42, 395-400 (1998).

56. Raithel, G., Phillips, W. D. \& Rolston, S. L. Coherence decay of wave-packets in optical lattices. Phys. Rev. Lett. 81, 3615 (1998)

57. Haycock, D.L., Alsing, P. M ., Grondalski, J., Deutsch, I. H. \& Jessen, P. S. M esoscopic quantum coherencein an optical lattice. Phys. Rev. Lett. 85, 3365 (2000).

58. Brennen, G., Caves, C., Jessen, P. \& Deutsch, I. Q uantum logic gates in optical lattices. Phys. Rev. Lett. 82, 1060-1063 (1999). 
59. Jaksch, D., Briegel, H. J., Cirac, J. I., Gardiner, C. W. \& Zoller, P. Entanglement of atoms via cold controlled collisions. Phys. Rev. Lett. 82, 1975-1978 (1999).

60. Jaksch, D. et al. Fast quantum gates for neutral atoms. Phys. Rev. Lett. 85, 2208-2211 (2000).

61. DeM ille, D. Quantum computation with trapped polar molecules. Preprint quant-ph/0109083 at http://xxx.lanl.gov (2001).

62.Jaksch, D., Bruder, C., Cirac, J. I., Gardiner, C. W. \& Zoller, P. Cold bosonic atoms in optical lattices Phys. Rev. Lett. 81, 3108-3111 (1998).

63. Spekkens, R. W. \& Sipe, J. E. Spatial fragmentation of a Bose-Einstein condensatein a double-well potential. Phys. Rev. A 59, 3868-3877 (1999).

64. Orzel, C., Tuchman, A. K., Fenselau, M. L., Yasuda, M. \& Kasevich, M. A. Squeezed states in a Bose Einstein condensate. Science 291, 2386-2389 (2001).

65. Greiner, M ., M andel, O., Esslinger, T., H änsch, T. W. \& Bloch, I. Quantum phasetransition from a superfluid to a M ott insulator in a gas of ultracold atoms. Nature 415, 39-44 (2002).

66. Cirac, I. \& Zoller, P. A scalable quantum computer with ions in an array of microtraps. Nature $\mathbf{4 0 4}$ $579-581(2000)$

67. M onroe, C., M eekhof, D. M., King, B. E. \& Wineland, D. J. A Schrödinger cat superposition state of an atom. Science 272, 1131-1136 (1996).

68. Bennett, C. H. et al. Purification of noisy entanglement and faithful teleportation via noisy channels. Phys. Rev. Lett. 76, 722-725 (1996).

69. Briegel, H.-J., Dür, W., Cirac, J. I. \& Zoller, P. Q uantum repeaters: the role of imperfect local operations in quantum communication. Phys. Rev. Lett. 81, 5932-5935 (1998).

70. Cirac, I., Zoller, P., Kimble, H . \& M abuchi, H. Quantum statetransfer and entanglement distribution among distant nodes in a quantum network. Phys. Rev. Lett. 78, 3221-3224 (1997).

71. Law, C. \& Kimble, H. Deterministic generation of a bit-stream of single-photon pulses. J. M od. O pt. 44, 2067-2074 (1997).

72. van Enk, S., Cirac, I. \& Zoller, P. I deal quantum communication over noisy channels, a quantum optical implementation. Phys. Rev. Lett. 78, 4293-4296 (1997).

73. Ye, J., Vernooy, D. W. \& Kimble, H. J. Trapping of singleatoms in cavity QED. Phys. Rev. Lett. 83, 4987-4990 (1999).

74. Hood, C. J., Lynn, T. W., D oherty, A. C., Parkins, A. S. \& Kimble, H. J. Theatom-cavity microscope: singleatoms bound in orbit by single photons. Science 287, 1447-1453 (2000).

75. Pinske, P., Fischer, T., M aunz, P. \& Rempe, G. Trapping an atom with single photons. Nature 404, 365-368 (2000).

76. Doherty, A., Lynn, T. W., Hood, C. J. \& Kimble, H. J. Trapping of singleatoms with singlephotons in cavity QED. Phys. Rev. A 63, 013401-1-013401-24 (2001).
77. Guthöhrlein, G. R. Keller, M. Hayasaka, K. Lange, W. \& Walther, H. A singleion as a nanoscopic probe of an optical field. Nature414, 49-51 (2001).

78. Liu, C., Dutton, Z., Behroozi, C. H. \& Hau, L. V. Observation of coherent optical information storage in an atomic medium using halted light pulses. Nature 409, 490-493 (2001).

79. Phillips, D. F., Fleischhauer, A., M air, A., Walsworth, R. L. \& Lukin, M. D. Storage of light in atomic vapor. Phys. Rev. Lett. 86, 783-786 (2001).

80. Budker, D., Kimball, D. F., Rochester, S. M . \& Yashchuk, V. V. Nonlinear magneto-optics and reduced group velocity of light in atomic vapor with slow ground staterelaxation. Phys. Rev. Lett. 83, 1767-1770 (1999).

81. Lukin, M \& \& Imamoglu, A. Controlling photons using electromagnetically induced transparency. Nature 413, 273-276 (2001)

82. Fleischhauer, M . \& Lukin, M. Dark-state polaritons in electromagnetically induced transparency. Phys. Rev. Lett. 84, 5094-5097 (2000)

83. M air, A., Hager, J., Phillips, D. F., Walsworth, R. L. \& Lukin, M . D. Phase coherenceand control of stored photonic information. Preprint quant-ph/0108046 at http://xxx.lanl.gov (2001).

84. Lukin, $M$. et al. Dipole blockadeand quantum information processing in mesoscopic atomic ensembles. Phys. Rev. Lett 87, 037901-1-037901-4 (2001)

85. Hald, J., Sørensen, J. L., Schori, C. \& Polzik, E. S. Spin squeezed atoms: a macroscopic entangled ensemble created by light. Phys. Rev. Lett. 83, 1319-1322 (1999).

86. Kuzmich, A., M andel, L. \& Bigelow, N. Generation of spin squeezing via continuous quantum nondemolition measurement. Phys. Rev. Lett. 85, 1594-1597 (2000).

87. Julsgaard, B., Kozhekin, A.\& Polzik, E. Experimental long-lived entanglement of two macroscopic objects. Nature 413, 400-403 (2001)

88. Duan, L.-M ., Lukin, M . D., Cirac, J. I \& \& Zoller, P. Long-distancequantum communication with atomic ensembles and linear optics. N ature 414, 413-418 (2001).

89. Wolf, F. A. Taking theQ uantum Leap (H arper and Row, San Francisco, 1981).

90.Zurek, W. H. Decoherence and thetransition from quantum to classical. Phys. Today $\mathbf{4 4 , 3 4 - 4 4}$ (1991).

91. Braunstein, S. L., D'Ariano, G. M ., M ilburn, G. J. \& Sacchi, M . F. Universal teleportation with a twist. Phys. Rev. Lett. 84, 3486-3489 (2000).

\section{Acknowledgements}

This work is supported by the US National Security Agency and Advanced Research and

Development Activity under an Army Research Office contract, and by the National

Science Foundation ITR programme. 